\title{
Population et environnement : des liaisons incertaines
}

\author{
Aicha Ouharon \\ Économiste, CIRED, Jardin tropical, 45 bis avenue de la Belle Gabrielle, 94736 Nogent-sur-Marne, France
}

La prise de conscience récente de la fragilité de l'environnement a relancé le débat autour de la théorie malthusienne de la population. L'auteur, en tant qu'économiste, tout en exposant les prolongements actuels de cette confrontation entre malthusiens et populationnistes, se refuse prudemment à trancher entre les deux théories, par absence de validation empirique à un niveau global. Le point de vue du démographe qui l'accompagne en commentaire, montre, à partir de références différentes, la difficulté méthodologique de modéliser les relations complexes entre écologie et démographie. Devant les difficultés communes d'appréhender globalement les logiques entre population et environnement dans les deux disciplines, on peut se demander si les approches locales de cette problématique ne sont pas les plus fécondes.

La Rédaction

\section{Mots-clés : \\ dynamique ; démographie; développement; environnement; incertitude}

\section{Keywords: dynamics, demography, development, environment, uncertainty}

\begin{abstract}
Résumé - Un traitement théorique cohérent du lien entre dynamique démographique et changement environnemental montre que cette relation demeure incertaine. Bien que l'on ait souvent mis l'accent sur la relation négative et significative entre ces deux variables depuis la fin des années 1970, plusieurs difficultés économétriques suggèrent qu'il puisse s'agir d'un artefact statistique. Les difficultés d'une validation empirique incontestable de la relation entre croissance démographique et croissance économique créent ainsi une situation de controverse, où il est difficile de quantifier les liens entre les déterminants du changement environnemental, et donc de trancher en toute rigueur sur le choix d'un modèle théorique plutôt que sur un autre.
\end{abstract}

\begin{abstract}
Population and environment: uncertain connections. A consistent theoretical assessment of the links between demographic and environmental dynamics shows that these links remain uncertain. Two opposite paradigms can put forward in this debate. A first paradigm considers that technical change is exogenous to the economic system. Due to decreasing outputs, the capital dilution effect and negative externalities on the environment, rapid demographic growth has a negative effect on both economic growth and the environment. On the other hand, the second paradigm assumes that, in the long term, economic growth is stimulated by population growth, thus inducing an improvement in environmentally friendly technologies, in conformity with the growth models based on endogenous technical change. Although, emphasis has often been put since the late 1970s on the negative and significant link between these two variables, several econometric difficulties suggest that this may prove to be a statistical artifact. The difficulties of an irrefutable empirical validation of the link between demographic and economic growths create a situation of controversy which make it difficult to quantify the relation between the determinants of environmental change and therefore to rigorously select one out of several theoretical models.
\end{abstract}

Depuis quelques décennies, des problèmes environnementaux d'un type nouveau ont émergé : aux problèmes de pollution très localisés (pollution des rivières, érosion du sol, etc.), et pour lesquels les sources et les effets étaient clairement déterminés, s'ajoutent

Auteur correspondant : ouharon@centre-cired.fr

A. Ouharon est maître de conférences à l'université de Paris 8 en sciences économiques et chercheure associée au Centre international de recherche sur le développement et l'environnement. des problèmes de nature plus globale (augmentation de l'effet de serre, perte de biodiversité, etc.). Dans ce cadre, la concomitance de menaces graves sur les grands équilibres de la planète et d'une croissance démographique mondiale sans précédent rendent incontournable l'analyse des conséquences démographiques sur l'environnement global. Or, dans les travaux économiques et démographiques portant sur l'environnement global, le lien entre démographie et économie demeure 
implicite et peu exploré. Une analyse économique adéquate de cette relation pourrait apporter un éclairage utile à ce débat.

Le but de cet article est de faire le point sur l'analyse théorique des conséquences de la croissance démographique sur le changement environnemental au niveau global et de sa validation empirique. Bien que les interactions entre le démographique, l'économique et le vivant soient complexes, il est possible d'organiser cette complexité autour de fortes postures opposées en la ramenant à des mécanismes démographiques et économiques de base bien identifiés. Par ailleurs, si l'on a souvent mis l'accent sur la relation négative et significative entre ces deux variables depuis la fin des années 1970, plusieurs difficultés économétriques suggèrent qu'il peut s'agir d'un artefact statistique, et une controverse durable s'est développée sur les arguments théoriques avancés pour expliquer cette relation.

Nous allons, dans un premier temps, situer les modèles à propriétés contrastées les uns par rapport aux autres en focalisant l'attention sur quelques paramètresclés, de manière à comprendre ce qui nourrit la controverse. Nous examinerons, dans un deuxième temps, les raisons expliquant la persistance de cette controverse en mettant en évidence les difficultés économétriques d'une validation empirique de la relation entre population et croissance économique.

\section{Une controverse jusqu'à présent ouverte}

Le niveau d'échelle global pose une difficulté fondamentale à la validation empirique de la relation entre population et croissance économique (Dasgupta, 1995; Keyfitz, 1991). Celle-ci rejaillit sur la relation populationcroissance économique-environnement, puisque la relation population-croissance économique constitue le premier maillon de la chaîne. Pour contourner cet obstacle, certains démographes et économistes ont souvent utilisé une identité comptable, où l'on décompose le changement environnemental en différents déterminants indépendants pour établir le poids relatif de chacun d'eux dans ce changement ${ }^{1}$ (Bongaarts, 1992 ; Birdsall, 1994; Lutz, 1994). Cette méthode est contestable, car elle se contente d'étudier les conséquences de la croissance démographique sur le changement environnemental, toutes choses égales par ailleurs, en omettant ainsi par construction la prise en compte des liens théoriques qui déterminent les interactions entre la population, la croissance économique et le progrès technique.

${ }^{1} I=I / C \times C / Y \times Y / C \times P$, où $I=$ impact environnemental $; I / C=$ impact environnemental par unité de consommation d'énergie; $C / Y=$ consommation d'énergie par unité du PIB; $Y / P=$ PIB par tête $; P=$ population.
Dès qu'on prend en compte ces interactions, on débouche sur la réactivation de différentes explications concurrentes du lien entre population et ressources naturelles. Du fait des rendements décroissants (Malthus, 1986a), de l'effet de dilution du capital (Solow, 1956) et des externalités négatives sur les ressources naturelles (Solow, 1974), une première approche considère que la croissance démographique rapide a une conséquence négative sur la croissance économique et sur les ressources naturelles. Une deuxième perspective, au contraire, considère que, sur le long terme, la croissance de la population stimule la croissance économique et induit une amélioration de la technologie du point de vue environnemental (Simon et Steinman,1992).

\section{L'explication malthusienne}

Tout en considérant l'état stationnaire comme une hypothèse éloignée dans le temps, les économistes classiques (en l'occurrence Malthus) considèrent les ressources naturelles comme un facteur limitant de la croissance démographique et économique.

Malthus estime que le progrès technique est une variable exogène et que le facteur démographique est en partie dépendant de la croissance économique. En effet, c'est la population qui s'adapte ex ante à la capacité productive. L'accroissement du produit total, qui a lieu grâce au progrès technologique, se trouve complètement absorbé, au bout d'un certain délai, par l'accroissement démographique. Ce modèle se base sur une fonction de production à rendements décroissants. En supposant un facteur de production fixe (la ressource naturelle), on s'attend à ce qu'une population plus nombreuse implique une production plus importante, mais selon une progression moindre que celle de la population, d'où une baisse du produit par tête.

Malthus postule que la population croît plus rapidement que les subsistances. Il affirme qu'il est nécessaire de maintenir en équilibre les deux pouvoirs multiplicateurs de la population et des subsistances qui suivent des évolutions inégales. Alors, un frein positif s'impose et limite la population. La croissance de la population baisse la production par tête, ce qui conduit à augmenter le taux de mortalité. Il y a donc une crise récurrente qui se produit pour réguler, par la hausse du taux de mortalité, l'évolution inégale des variables population et ressources naturelles. Dans l'analyse de Malthus, la ressource naturelle apparaît ainsi explicitement au sens où le facteur de production fixe est un facteur environnemental (Malthus, ibid.).

Dans son deuxième essai, plus interdisciplinaire, Malthus va au-delà de ce modèle biologique en formulant la notion de frein de prudence (Malthus, 1986b). Celui-ci est, certes, une forme de frein préventif, mais 
opérant dans une variété plus large de circonstances. La réponse démographique aux variations du salaire peut être conçue comme un cas de rationalité limitée ${ }^{2}$. Les êtres humains tendent à se reproduire toutes les fois qu'il y a hausse du niveau de vie. Mais, si les individus ont comme objectif d'améliorer leur niveau de vie, ils vont choisir de retarder leur entrée en union ainsi que le moment de reproduction. Malthus décrit les individus rationnels comme étant capables d'anticiper un meilleur niveau de vie. Sur un horizon temporel significatif, le taux d'accroissement de la population devient négativement (au lieu de positivement) corrélé au niveau du salaire réel. $C^{\prime}$ est en ce sens que le frein de prudence offre un aspect analytique nouveau au débat sur la population (Malthus, 1986c).

\section{De Malthus à Solow}

Malthus, comme les autres économistes classiques, considère les ressources naturelles (en l'occurrence la terre) comme un facteur limitant de la croissance. Son inquiétude porte sur l'épuisement de ces ressources et ses conséquences sur la croissance de la richesse. Elle est levée par les néoclassiques grâce à l'hypothèse de substituabilité entre capital artificiel et capital naturel, le processus d'investissement et l'amélioration de l'efficacité dans l'usage des ressources naturelles.

La durabilité faible ${ }^{3}$ proposée par Solow (1993) répond à l'exigence de préserver de façon indéfinie la capacité productive des économies, qui ne se limite plus seulement aux équipements productifs, comme dans le modèle initial, mais comprend tous les actifs qui contribuent au bien-être, y compris le capital naturel.

Solow (1974) montre que l'introduction de ressources naturelles ne modifie pas radicalement les résultats qu'il avait obtenus dans le modèle initial (sans ressources naturelles), dès lors qu'il n'y a pas de limite physique à la croissance. Le sentier optimal est un sentier à consommation constante le long duquel l'investissement en capital manufacturé compense exactement l'épuisement de la ressource naturelle ${ }^{4}$. C'est le stock total (manufacturé et naturel) qui est maintenu constant le long du sentier optimal, seule sa composition se modifie dans le sens de la baisse du capital naturel.

Dans le modèle de Solow, l'effet négatif ne concerne que la relation entre taux de croissance de la population

\footnotetext{
2 En ce sens que les individus ne disposent pas de toutes les informations pour anticiper parfaitement leur niveau de vie.

${ }^{3}$ La durabilité faible se réfère à une typologie proposée par K. Turner (Godard, 2004), partant du moins exigeant (durabilité faible) au plus exigeant (durabilité forte) du point de vue de la protection de l'environnement.

4 Ce résultat est intuitif du moment que l'on considère le stock de capital, manufacturé et naturel, comme un tout.
}

et niveau de revenu : $c^{\prime}$ est l'effet de dilution de capital. Le modèle implique qu'il y a baisse transitoire du produit par tête lorsque la population passe d'un niveau à un niveau supérieur. Ensuite, si la population se stabilise à nouveau, on retrouve le rapport capital/travail initial, et tout effet d'échelle disparaît. Mais, si la population se met à croître sans interruption, alors le retard du capital par rapport à la population devient permanent, et il y a stabilisation du rapport capital/travail et de la production par tête à un niveau inférieur (Blanchet, 1991a).

La relation est donc négative si l'économie se trouve en dehors de l'état stable suite à un choc externe causé par la croissance démographique. En effet, si on compare les différents états d'équilibre dynamiques possibles, qui sont déterminés par la propension à épargner et le taux de croissance démographique, on constate que le revenu par tête d'équilibre - pour un taux de progrès technique donné - est d'autant plus élevé que la dotation en capital de chaque travailleur est grande. En conséquence, le revenu par tête d'équilibre sera d'autant plus élevé que : (i) la propension à épargner sera forte ; (ii) la croissance de la population et de la main-d'œuvre sera faible ${ }^{5}$.

$\mathrm{Et}$, dans un cas de défaillance du marché, c'est-à-dire de mauvaise définition des droits de propriété sur les ressources naturelles, la croissance rapide de la population est un facteur accélérateur de la dégradation environnementale consécutive à l'utilisation des ressources naturelles en libre accès. Dans ce contexte, le niveau d'utilisation de la ressource naturelle augmente, toutes choses égales par ailleurs, corrélativement à l'accroissement de la taille de la population y ayant accès. La conclusion valable dans le modèle initial (sans ressources naturelles) l'est aussi ici. Si l'on se met dans la position de pouvoir décider de la taille de la population, la dotation en ressources naturelles de chaque individu sera d'autant plus élevée que la croissance démographique sera faible. Une maîtrise de la croissance démographique rapide est souhaitable.

Dans la pensée malthusienne comme dans celle de Solow, c'est l'être humain qui décide des variables d'ajustement de l'offre limitée des ressources naturelles à la demande croissante de biens de consommation et de production. On est dans une optique de progrès sous contraintes des ressources naturelles limitées, mais où les êtres humains peuvent maîtriser la trajectoire de leur évolution en tenant compte des contraintes initiales. Les politiques publiques gardent ainsi leur liberté d'action sur tel ou tel choix de consommation, de production ou de niveau de population. Au sein de cette première perspective, la notion, issue de la biologie, de population limite se distingue des modèles de Malthus et de Solow.

\footnotetext{
5 Dans la démarche néoclassique, l'élévation du revenu par tête provient donc soit du capital par unité de travail (à progrès technique constant), soit du déplacement de la fonction de progrès technique (à intensité capitalistique donnée).
} 


\section{Le cercle vertueux de la population}

Le modèle de Solow ne prend pas en compte l'effet possible de l'élargissement de l'échelle de production, c'est-à-dire l'existence de rendements croissants. L'analyse s'opère uniquement en termes de taux de croissance des facteurs ${ }^{6}$. Et cela, pour une raison simple, qui tient à la liaison entre technique de production et théorie de la répartition. Pour que le postulat de rémunération des facteurs à leur productivité marginale soit respecté, il faut que les rendements à l'échelle soient constants. La nécessité de sauvegarder les bases techniques de la théorie de la répartition explique également la nature du concept du progrès technique utilisé. Pour que le progrès technique ne soit pas un facteur à rémunérer, il est alors considéré comme un deus ex machina, dont les effets améliorent l'efficacité des "vrais » facteurs de production. Cette autonomie exige que le progrès technique soit totalement indépendant de l'évolution démographique. Elle implique aussi que la croissance démographique soit le plus faible possible, et donc que l'on puisse répartir les gains de l'innovation sur le moins d'individus possible.

Si Malthus et Solow ne reconnaissent aucune influence de la croissance de la population sur le progrès technique, les "populationnistes » en font la pièce mâ̂tresse de leur démonstration (Birdsall, 1988). Selon eux, l'histoire économique montre clairement que la rareté des ressources naturelles, induite par la croissance de la population, stimule des changements significatifs de la technique. La croissance démographique donne lieu à des coûts d'ajustement dans le court terme; mais, dans le long terme, en s'étendant à tout le système économique et social, la rareté des ressources naturelles se révèle source d'innovation. La baisse de la croissance économique induite par la contrainte des ressources naturelles - la trappe malthusienne - est levée par le mécanisme de l'innovation et du progrès technique. Nous retenons ici notamment une manière « $\mathrm{d}$ 'endogénéiser » le progrès technique : celle considérée par Simon et Steinman (1992).

Pour Simon (1985), les ressources naturelles, à l'instar d'autres biens, sont affectées d'un prix qui représente théoriquement la mesure économique de leur rareté. L'augmentation persistante des prix sur le long terme est un signal suffisant pour permettre la gestion efficace d'une ressource naturelle et sa substitution progressive par d'autres biens moins coûteux. Les ressources naturelles, les biens de consommation et de production trouvent leur meilleure allocation par l'entremise du marché. À cet égard, Simon (1989) note la baisse séculaire des prix des matières premières et des ressources

\footnotetext{
6 Cela est possible grâce au raisonnement «toutes choses égales par ailleurs », qui permet de distinguer entre rendement et progrès technique. Le rendement se calcule à l'aide d'un déplacement homothétique de tous les facteurs, à technique constante.
}

énergétiques. Cette baisse des prix est la conséquence du progrès technique qui, sous l'impulsion de l'accroissement démographique, permet une substitution toujours plus grande entre le capital naturel et le capital manufacturé. La rareté et le progrès technique s'influencent mutuellement au travers des hausses et des baisses des prix successives qu'induisent augmentation de la demande et processus de substitution. Dès lors que les prix relatifs changent, les effets qui en résultent vont au-delà des mécanismes de la simple substitution qui est prévue par les modèles microéconomiques. Les changements de prix provoquent des transformations extensives des systèmes technologiques (produits et processus) qui rendent difficilement réversible un usage plus efficace des ressources naturelles.

Bien évidemment, d'autres manières d'endogénéiser le progrès technique existent. Ester Boserup (1981) est la plus souvent associée à cette perspective théorique. Sa thèse centrale consiste à dire que la croissance démographique à court terme, en raison des rendements décroissants du travail, provoque une baisse du revenu, qui stimule le processus d'innovation. L'adoption des nouvelles techniques permet de contrecarrer la loi des rendements décroissants, et donc la baisse de la production par tête. Sur le long terme, la fonction de production s'ajuste vers le haut et la population accrue se trouve avec un niveau de vie supérieur à celui de la situation initiale.

La critique fondamentale que $l^{\prime}$ on peut apporter à ces deux modèles est la non-prise en compte de l'irréversibilité d'usage des ressources naturelles. Comme l'irréversibilité est associée à une défaillance du système de marché, cette dernière ne peut être corrigée par une modification du système de prix : la valeur de quasi-option ${ }^{7}$ doit être rajoutée à la liste des avantages à inscrire au bilan coûts-avantages des décisions flexibles. En d'autres termes, les potentialités d'exploiter des informations ultérieures sur les états du monde sont prises en compte. Le choix de préserver un certain bien environnemental est justifié par la possibilité d'explorer l'information de stratégies de choix alternatifs dans le futur. Par ailleurs, Simon et Boserup ne font aucune différence entre les caractéristiques des ressources naturelles et celles du capital manufacturé. Alors que ce dernier est toujours capable de variation symétrique - il peut être diminué ou accru -, les ressources naturelles sont sujettes aux irréversibilités - elles peuvent être diminuées mais rarement augmentées, notamment si un certain seuil dans la baisse est franchi, qui les conduit à l'épuisement. Les ressources naturelles et le capital ne sont des substituts qu'en mesure limitée. Les deux auteurs, ne prenant en compte à long terme ni les effets de seuil, ni les irréversibilités, ni les valeurs d'options, ni les limites techniques à la substitution,

7 La valeur de quasi-option est la valeur espérée de l'information pour un décideur confronté à un problème de décision en incertain avec possibilité d'acquisition d'information. 
ni l'incertitude concernant l'évolution future du progrès technique, considèrent que toute augmentation de la rareté d'une ressource naturelle stimule l'innovation (ou l'adoption) et le phénomène de substitution.

Aussi, la façon d'envisager les rapports population/ressources naturelles du modèle de Simon n'est pas à l'abri de la critique. Néanmoins, elle permet d'entrevoir une autre logique que celle du modèle malthusien et de mettre en évidence les points faibles de celui-ci. Voyons, à présent, pourquoi l'appréhension de la relation entre population et environnement global se heurte actuellement à la difficulté d'une validation empirique non contestable.

\section{Les difficultés de la validation empirique}

La persistance de la controverse théorique est due au fait que la relation entre population et environnement global n'est pas directe, mais médiatisée par la croissance économique. Or, au niveau global, on se heurte à la difficulté de la validation empirique des rapports entre population et croissance économique. Depuis la fin des années 1970 est identifiée une relation entre croissance démographique et croissance économique, tout à fait significative et fortement négative selon les critères statistiques habituels. Cependant, cette validation empirique demeure contestable, car on ne sait trancher sur le fait de savoir s'il s'agit d'un réel changement ou d'un artefact statistique (Blanchet, 1991b). Cinq difficultés économétriques sont à l'origine de cette indétermination empirique :

1. Dans la spécification des modèles, on peut omettre des variables déterminantes sur la croissance économique, ce qui peut biaiser l'évaluation des effets de la croissance démographique sur celle-ci. Deux éléments peuvent expliquer cette difficulté : d'une part, on ne dispose pas encore d'un modèle empirique robuste dans le domaine de la croissance économique; d'autre part, il existe des facteurs institutionnels difficilement mesurables.

2. Des problèmes de multicolinéarité surgissent si l'on introduit davantage de variables pour former l'ossature d'une structure théorique. Par exemple, les taux de natalité élevés induisent, 15 ans plus tard, des taux de croissance élevés de la population active. Ces taux de natalité élevés étant positivement corrélés entre eux dans le temps, la régression ne permet pas d'isoler l'influence d'un seul taux de natalité sur le taux de la population active. En outre, plusieurs facteurs du processus du développement agissent vite et sont, par conséquent, corrélés : les taux d'éducation augmentent, le travail se réduit dans le secteur agricole, les taux de natalité baissent, et la mortalité aussi. Même si elle estime correctement les effets, toutes choses égales par ailleurs, la régression est incapable de rendre compte de l'imbrication entre les variables corrélées. Dans les modèles qui étudient le lien entre croissance démographique et croissance économique, maintenir trop de variables colinéaires conduit à des estimations statistiques imprécises et remet en question la signification statistique de ces modèles.

3. Le traitement approprié de l'hétéroscédasticité ${ }^{8}$ est souvent ignoré. En effet, on peut se demander si les régressions avec pondération, qui attribuent plus de poids à la croissance démographique d'un grand pays, ne risquent pas de lui attribuer une influence indue. Inversement, on peut se demander si les régressions sans pondération n'attribuent pas un poids plus important à la croissance démographique des petits pays. Les études évaluent rarement les impacts de schémas de pondération alternatifs, car ceux-ci peuvent significativement altérer les coefficients estimés.

4. Le biais de simultanéité pose les problèmes les plus sérieux à la relation étudiée. Il vient de ce que la croissance économique détermine autant la croissance démographique qu'elle est perturbée par elle (McNicoll, 1984). Deux choses attestent l'existence de ce biais : (a) d'une part, le bouclage peut quand même être relativement rapide, se déroulant sur une échelle de temps de l'ordre de la période de cinq ou dix ans sur laquelle sont mesurés les taux de croissance, ce qui est indéniable s'agissant d'une des composantes de la croissance démographique, le mouvement migratoire; (b) d'autre part, la croissance économique est un phénomène autocorrélé. Même si c'est la croissance économique passée qui affecte la croissance démographique courante, cette dernière peut se retrouver liée de facto à la croissance économique courante du simple fait que les pays qui ont connu une croissance rapide à une certaine période ont des chances de voir cette croissance perdurer à la période suivante.

5. Des problèmes peuvent surgir dès lors que l'on doit composer avec l'évaluation dynamique des conséquences de la croissance démographique. En effet, les fonctions économiques d'un individu varient durant son cycle de vie. Dans le court terme, une naissance présente vraisemblablement une charge économique, mais contribuera 15 ans après à la richesse de la société. Les études empiriques transversales ne distinguent pas entre les conséquences économiques négatives des naissances présentes et les conséquences économiques positives des naissances passées.

Le résumé d'évaluation de Lee (1983) est instructif : «Les études à coupe transversale n'ont pas fourni le résultat espéré : un portrait sommaire et stylisé des conséquences de la croissance rapide de la population; à moins que, certes, l'absence de résultats significatifs soit en ellemême un résultat. »

8 L'hétéroscédasticité désigne le cas où la variance du terme d'erreur n'est pas constante pour toutes les valeurs de la variable indépendante. 


\section{Conclusion}

Localement, quand l'être humain dégrade la terre ou pollue une rivière, suite à la pression de la population, tout est relativement simple. Si des villageois règlent leur action en négligeant les nuisances causées aux autres, le phénomène est physiquement perceptible et les acteurs, bien identifiés. La protection de l'environnement se justifie, car son coût reste inférieur aux satisfactions qu'elle procure. Certes, les écologistes peuvent reprocher à l'économie de réduire le problème à la sphère marchande. Cependant, le calcul économique peut se prévaloir du succès du principe pollueur-payeur, inspirant des règles permettant de concilier intérêt individuel et intérêt collectif.

En revanche, les problèmes d'environnement global sont d'une nature tout à fait différente. Dans ce cas, l'incertitude des théories scientifiques concernant les phénomènes de pollution (nature et conséquences à long terme) se double d'une controverse relative à la nature de la liaison entre la population, le développement économique et l'environnement. Ensuite, les horizons temporels en jeu (le très long terme) accentuent fortement le degré d'incertitude scientifique au sujet des évolutions environnementales. On a tout autant à réguler des comportements qu'à coordonner des anticipations concurrentes portant sur des visions du monde. Ces anticipations sont à présent inconciliables, car les causes, la dynamique d'évolution et les conséquences potentielles sont incertaines et controversées.

\section{Références}

Birdsall, N., 1988. Economic approaches to population growth, in Chenery, H., Srinivasan, T.N., (Eds), Handbook of Development Economics I, Amsterdam, North Holland, 478-542.

Birdsall, N., 1994. Another look at population and global warming, in United Nations, Population, Environment and Development, New York, United Nations, 39-54.

Blanchet, D., 1991a. Modélisation économique, conséquences économiques des évolutions démographiques, Paris, INED/PUF.

Blanchet, D., 1991b. Estimer l'effet de la croissance démographique sur la croissance économique dans les PVD : problèmes méthodologiques, in Tapinos, G., Blanchet, D., Horlacher, D.E. (Eds), Conséquences de la croissance démographique rapide dans les pays en développement, Paris, INED, 57-86.
Bongaarts, J., 1992. Population growth and global warming, Population and Development Review, 18, 2, 299-320.

Boserup, E., 1981. Population and Technological Change: A Study of Long-Term Trends, Chicago, University of Chicago Press.

Dasgupta, P., 1995. The population problem: theory and evidence, Journal of Economic Literature, XXXIII, 1879-1902.

Godard, O., 2004. La pensée économique face à la question de l'environnement, Cahiers du Laboratoire d'économétrie, 25, 1-30.

Keyfitz, N., 1991. Population and development within the ecosphere: one view of the literature, Population Index, 57, 1, 5-22.

Lee, R., 1983. Economic consequences of population size, structure and growth, Newsletter International Union for the Scientific Study of Population, 17, 43-59.

Lutz, W., 1994. Population and environment - What do we need more urgently: better data, better models or better questions?, in Zaba, B., Clark, J. (Eds), Environment and Population Change, Liège, International Union for the Scientific Study of Population et Ordina Editions, 47-62.

Malthus, T.R., 1986a. An Essay on the Principle of Population [1798], in Wrigley, E.A., Souden, D. (Eds), The Works of Thomas Robert Malthus, London, Pickering \& Chatto Publishers, 1 , 1-139.

Malthus, T.R., 1986b. An Essay on the Principle of Population [1826], in Wrigley, E.A., Souden, D. (Eds), The Works of Thomas Robert Malthus, London, Pickering \& Chatto Publishers, 2 \& 3, 1-711.

Malthus, T.R., 1986c. Principles of Political Economy [1836], in Wrigley, E.A., Souden, D. (Eds), The Works of Thomas Robert Malthus, London, Pickering \& Chatto Publishers, 5 \& 6, 1-327.

McNicoll, G., 1984. Consequences of rapid population growth: an overview and assessment, Population and Development Review, 10, 2, 177-240.

Simon, J.L., 1985. L'Homme, notre dernière chance : croissance démographique, ressources naturelles et niveau de vie, Paris, PUF.

Simon, J.L., 1989. On aggregate empirical studies relating population variables to economic development, Population and Development Review, 15, 2, 323-333.

Simon, J.L., Steinman, G., 1992. Population, natural resources, and the long-run standard of living, in Simon, J.L. (Ed.), Population and Development in Poor Countries: Selected Essays, Princeton, Princeton University Press, 89-121.

Solow, R., 1956. A contribution to the theory of economic growth, Quarterly Journal of Economics, 70, 1, 65-94.

Solow, R., 1974. Intergenerational Equity and Exhaustible Resources, Review of Economic Studies, Special issue, 29-46.

Solow, R., 1993. An Almonst Practical Step Toward Sustainability, Resources Policy, 19, 3, 162-72. 